Revue européenne des sciences sociales

European Journal of Social Sciences

XXXIX-121 | 2001

L'acteur. Un concept sur la scène des sciences sociales

\title{
Acteur ou agent : les usages de la règle
}

Mark Hunyadi

\section{OpenEdition}

Journals

Édition électronique

URL : http://journals.openedition.org/ress/644

DOI : $10.4000 /$ ress. 644

ISSN : 1663-4446

Éditeur

Librairie Droz

Édition imprimée

Date de publication : 1 novembre 2001

Pagination : 15-30

ISBN : 2-600-00663-X

ISSN : 0048-8046

Référence électronique

Mark Hunyadi, «Acteur ou agent : les usages de la règle », Revue européenne des sciences sociales [En ligne], XXXIX-121 | 2001, mis en ligne le 11 décembre 2009, consulté le 30 avril 2019. URL : http:// journals.openedition.org/ress/644; DOI : 10.4000/ress.644 
Mark HUNYADI

\section{ACTEUR OU AGENT : LES USAGES DE LA RÈGLE}

1. Dans ce qui suit, j'aimerais poursuivre un double objectif qui m'a été inspiré par la lecture des réflexions de Jean-Pierre Gaudin: d'une part, proposer une définition des notions d'acteur et d'agent, mais en mobilisant non pas tant les éléments d'une ontologie personnelle formulée dans les termes d'une théorie de la liberté, comme il le fait légitimement, qu'une approche pragmatique centrée sur l'usage de la règle ( $\$ 14-17)$. Ceci nécessitera quelques considérations préalables sur la notion même de règle, qui sera le fil conducteur des présentes réflexions (§§6-13). D’autre part, il s'agira de dégager quelques implications normatives des distinctions qui auront été établies sur la base d'une telle «théorie critique de la règle », notamment dans la perspective démocratique de ce que j'appelle « la réappropriation de l'espace normatif par les acteurs sociaux eux-mêmes» (§§18-24).

2. Pour engager cette réflexion, et à titre de simple illustration préliminaire, j'aimerais partir de la constatation de sens commun que dans tous les domaines de la vie sociale s'affirme aujourd'hui la tendance lourde d'une exigence de participation émise par les concernés eux-mêmes: quel que soit le domaine de régulation (éthique, politique, juridique, économique), les gens veulent participer à l'élaboration des règles qu'ils utilisent ou dont ils dépendent: les utilisateurs des règles veulent aussi, en quelque manière, en être les auteurs. Cette participation à l'élaboration des règles apparaît aujourd'hui de plus en plus comme le véritable garant de la légitimité de celles-ci; c'est là un fait social qui mérite qu'on en dégage la signification normative.

3. Les exemples abondent, qui montrent - à rebours de ce qu'invoque la culture des élites, ou des experts - que la chose éthique est devenue chose publique. L'échange d'informations, la multiplication des points de vue, la délibération sont devenues inséparables du raisonnement puis de la prise de décision éthique - et ce fait-là me semble cristalliser un enjeu normatif de la plus haute importance, que je caractériserais comme la tendance à la réappropriation de l'espace normatif par les acteurs sociaux eux-mêmes. (Voir par exemple la «Conférence des citoyens » sur les organismes génétiquement modifiés en 1998 en France ${ }^{1}$ ). La tendance est multiforme: songeons par exemple au mouvement de déformalisation du droit; d'autres exemples abondent, du domaine micro-social

Voir la «Revue française de science politique», vol. 50, n 4-5, août-octobre 2000, pp. 779-809. Dans le même numéro, on lira avec intérêt, dans le même sens, l'article de Gilbert Larochelle sur "L'expérience des commissions sur l'avenir du Québec". 
(famille, quartier, entreprises - n'est-ce pas dans les entreprises qu'on a vu les premiers médiateurs? Il y en a aujourd'hui dans chaque organe de presse) au domaine macro-social (politiques de l'environnement aux niveaux régional, national, international, voire mondial; mais aussi politique démocratique au sens large ${ }^{2}$ ). Partout tend à s'imposer ce qu'il faut bien appeler le principe de participation, qui assure mieux que tout autre à la fois la légitimité et l'efficacité des décisions prises.

4. Mais c'est sans doute dans les mouvements de réaction à la mondialisation qu'il a récemment atteint une visibilité maximale : face à ce qui apparaît comme la nouvelle loi d'airain du développement historique, les acteurs sociaux dépossédés de leur pouvoir régulateur veulent se le réapproprier, et enrayer ce qu'ils ressentent comme une injuste colonisation de leur espace normatif. Cela veut dire que les acteurs sociaux ne se sentent précisément plus des acteurs, mais - selon une terminologie qui sera encore approfondie - des agents, des agents comme on dit «agent du KGB», c'est-à-dire au service d'un autre qu'eux, agents d'une puissance qu'ils n'ont pas choisie et qu'ils ne contrôlent pas, des agents qui sont en réalité agis par une force qu'ils ne maîtrisent pas.

5. Traditionnellement, le phénomène que je caractérise ici en termes de dépossession, confiscation, colonisation, est analysé à travers les concepts d'autonomie et d'hétéronomie, c'est-à-dire grosso modo en termes de liberté. Stratégiquement, cela conduit aux problèmes bien classiques de la définition de la liberté, individuelle ou collective, négative, positive, celle des Anciens et celle des Modernes, etc., et de la détermination de ses limitations par les contraintes sociales, naturelles, culturelles, génétiques, etc. Du point de vue de l'histoire des idées, on retrouve ici le thème de la modernité comme perte de liberté, thème bien connu depuis les analyses de Max Weber, qu'on retrouve encore chez Amartya Sen ou, plus récemment encore, chez Jean-Marc Ferry (dans son livre De la civilisation).

6. Mais c'est une autre ligne de lecture que j'aimerais proposer ici, une lecture qui prend pour fil conducteur non le thème de la liberté, mais celui de règle, et plus exactement celui d'obéissance à la règle. Il apparaît en effet que lorsque l'on parle, comme je l'ai fait, de pouvoir de régulation des acteurs sociaux, de réappropriation de l'espace normatif, de dépossession, de confiscation ou de colonisation de cet espace, on parle aussi, et à mon sens surtout, d'un certain rapport que les individus entretiennent aux règles, et du type d'emprise que celles-ci exercent sur ceux-là. Ceci suggère qu'une «théorie critique de la règle», qui distinguerait différents types de règles et différents modes d'obéissance à celles-ci - différents modes qui pourraient renvoyer, comme je vais essayer de le monter, soit à la pos-

Comme en écho au modèle délibératif et communicationnel de Habermas, Michel CrozIER ne dit pas autre chose: "Le dialogue social ne pourra se rétablir que par l'écoute, l'ouverture et la fin des monopoles de la représentation...: une nouvelle réflexion est indispensable sur les conditions d'une vie démocratique active dans un monde différent. La démocratie limitée à la représentation est insuffisante car elle donne trop d'importance, du moins dans le contexte français, au problème de l'accès aux représentants. Nous devons passer de la démocratie d'accès à la démocratie de la délibération". La crise de l'intelligence. Essai sur l'impuissance des élites à se réformer, Paris, InterEditions, 1995, pp. 61 et 62. 
ture de l'acteur, soit à la posture de l'agent-, pourrait aider à la fois à la compréhension de cette tendance lourde de notre temps, et à en tirer quelques leçons normatives.

7. Pour aborder la question de l'acteur social au fil d'une théorie de la règle, je partirai de quelques considérations que Wittgenstein a consacrées à cette notion dans un texte des années 30, paru en français à la fin des années $90^{3}$. Pour expliciter le double sens de l'expression «suivre une règle», Wittgenstein prend l'exemple du jeu. Que veut dire «règles» dans l'expression «règles d'un jeu »? Ce ne sont évidemment pas des règles au sens des lois de la nature que découvrirait celui qui observe un jeu, comme l'astronome les astres. Cela ne fait guère problème: les règles du jeu d'échecs ne sont pas de même nature que les lois de la gravitation. Mais la question philosophiquement intéressante surgit dès lors que l'on s'interroge sur les critères pertinents permettant de distinguer ces deux types de règles. Quelles sont donc les marques caractéristiques permettant de distinguer les deux sens de «règle», selon que l'on dit «le fou se déplace en diagonale», ou «les astres suivent une orbite elliptique »? Plusieurs candidats se proposent, mais en voici un certain nombre étroitement corrélés entre eux:

8. La différence la plus importante, celle dont découlent toutes les autres, est celle-ci: lorsque j'étudie le mouvement des astres pour savoir quelles règles ils suivent, je suis par définition confiné à la position d'observateur qui constate des régularités autorisant l'établissement d'une corrélation causale entre deux événements. En conséquence - et c'est là le point capital -, la position d'observateur m'oblige, en toute rigueur, à ne pouvoir formuler que des hypothèses: «Toute explication est une hypothèse », dit lapidairement Wittgenstein, soulignant par là non pas tant le caractère incertain des explications causales que le caractère par nature conjectural de toutes les explications qui visent à dire quelque chose du monde. Ainsi, les règles énoncées à propos des événements du monde sont-elles des régularités qui ne peuvent valoir que comme hypothèses explicatives.

9. Tout autres sont les règles au sens du jeu d'échecs. Lorsque je dis que le fou se déplace en diagonale, je ne formule pas du tout une hypothèse sur la relation causale entre deux événements; je ne dis pas du tout quelque chose sur le monde, je ne décris pas une régularité: j'énonce la prescription d'un usage déterminé d'une pièce du jeu d'échecs. Cette prescription ne résulte pas d'une observation anthropologique («dans tous les cas que j'ai observés, les hommes déplacent le fou en diagonale »), mais d'une convention instituée. Ainsi, si quelqu'un déplace son fou latéralement, je ne dirai pas que c'est là une nouvelle manière de jouer aux échecs, mais que celui-là ne sait pas jouer aux échecs. Telle est la force prescriptive des conventions, qui permettent de démarquer la pratique correcte de la pratique incorrecte; c'est là, très exactement, le rôle normatif des règles comprises comme conventions.

10. Mais ce qu'il y a de remarquable, c'est que cette force prescriptive, la règle comme convention ne peut l'acquérir que dans la perspective performative

\footnotetext{
Antonia SOULEZ (dir.), Dictées de Wittgenstein à Waismann et pour Schlick, 1, textes inédits (années 1930), Paris, P.U.F., 1997, p. 56. (Dorénavant: D).
} 
de celui qui suit la règle. Autant la position d'observateur condamne à la posture conjecturale, autant la position performative de celui qui suit la règle à la première personne est, à l'inverse, catégorique. Si j'énonce la règle que je suis, je n'émets pas une hypothèse de comportement, je ne décris pas une régularité ni n'avance une probabilité sur la cause de mon action, je dis, simplement, ce que je fais: je dis la prescription à laquelle j'obéis, et cette prescription est alors la raison de mon comportement. On quitte là le domaine des causes hypothétiques pour celui des raisons catégoriques. C'est pourquoi Wittgenstein dit à ce propos: «Une règle du jeu n'est donc pas une hypothèse qui sera satisfaite par les mouvements du joueur mais la règle que le joueur interrogé sur une règle donne en réponse» ${ }^{4}$. Notons encore une fois le changement de perspective qui préside à cette distinction entre deux types de règles: la règle comme hypothèse à satisfaire est établie du point de vue de la troisième personne, alors que la règle qui est celle que les joueurs suivent ne peut être connue que dans la perspective performative de la première personne, qui seule peut éprouver, sous forme de sentiment d'obligation, sa force prescriptive. C'est pourquoi Wittgenstein peut dire: «Raison et cause correspondent aux deux significations de l'expression "suivre une règle". La cause d'une action résulte d'une observation et cela de manière hypothétique, c'est-à-dire, de sorte que d'autres expériences puissent la confirmer ou la contredire. La raison est ce qui est indiqué comme tel $»^{5}$.

11. Ce nécessaire changement de point de vue a une raison simple: c'est que lorsqu'on observe un comportement, on ne peut jamais savoir quelle règle les acteurs suivent. Tout comportement observé est susceptible d'obéir à un nombre indéterminé (pour ne pas dire infini) de règles possibles. C'est cette constatation qui a justifié toute la littérature sur ce que l'on a appelé - faussement à mon avis «le paradoxe de Wittgenstein »: «Les mouvements du jeu satisfont à des règles infiniment nombreuses. Prenons l'exemple d'un homme qui écrit des chiffres: 2 , 4, 6, 8. Il aurait pu écrire également 100 chiffres et de là nous aurions pu déduire une loi de la nature suivie par l'activité de les écrire. Mais son activité aurait toujours satisfait un nombre infini de règles $»^{6}$. Ce que Wittgenstein veut semble-t-il dire, c'est que la suite $\{2,4,6,8\}$ inscrite sur un tableau noir peut certes satisfaire à la règle $\mathrm{y}=\mathrm{x}+2$, mais aussi à la règle selon laquelle $\mathrm{y}=\mathrm{x}+2$ vaut pour la première dizaine des entiers naturels, $\mathrm{y}=\mathrm{x}+3$ pour la deuxième dizaine ou pour toutes les dizaines suivantes, etc. Mais notons bien - et ceci émousse singulièrement l'usage hyperbolique du «paradoxe» décrit par Kripke ${ }^{7}-$ qu'un tel doute quant à la règle qui est réellement mise en œuvre ne peut principiellement surgir que du point de vue de la $3 \mathrm{e}$ personne. Pour la 1ère personne, son usage est, comme il a été dit, catégorique: car celui qui a écrit la suite sur le tableau noir peut, lui, dire sans doute possible quelle règle il a suivie. Il n'y a même que lui qui le puisse.

\footnotetext{
D 54 (C'est moi qui souligne).

D 56.

D 53.
}

Saul KRIPKE, Règles et langage privé, introduction au paradoxe de Wittgenstein, trad. de Thierry Marchaisse, Paris, Seuil, 1996. 
12. Une autre marque caractéristique de la distinction entre ces deux types de règles - une marque que Wittgenstein n'évoque pas du tout, mais qui à mon sens s'avérera décisive pour l'élaboration d'une théorie normative - est que les conventions, étant des institutions, sont critiquables, en un sens où les règlesrégularités ne le sont pas. On peut critiquer les règles instituées - c'est pourquoi elles se modifient sans cesse, elles sont sujettes à une évolution continue. Dans le cas des règles-régularités, ce n'est pas à proprement parler la règle que l'on critique, c'est la formulation de la règle, pour la mettre en meilleure adéquation avec le monde dont elle dit quelque chose. On voit bien à l'inverse qu'il n'y a aucun fait du monde qui puisse falsifier la règle selon laquelle le fou se déplace en diagonale, il n'y a aucune réalité superlative qui puisse démentir cette règle: on ne peut pas dire «Jusqu'à aujourd'hui, on croyait que le fou se déplace en diagonale, mais aujourd'hui, je peux prouver que c'était une erreur »; les règles du jeu d'échecs ne sont pas falsifiables en ce sens. Mais elles sont critiquables: on pourrait changer ses règles, comme on change les règles du football ou les taux d'imposition, et il y aurait alors une forme $d$ ' accord entre les utilisateurs de ces règles pour ce nouvel usage.

\section{En synthèse, on peut donc dire:}

- En ce qui concerne l'explication des actions humaines, le type de point de vue que l'on adopte sur l'agir (objectivant ou performatif) modifie le sens de l'expression «suivre une règle»:

- Du point de vue de l'observateur, on ne peut que produire des hypothèses explicatives, et la règle que l'on découvre alors est une règle au sens des régularités observées. La notion de cause est reliée de manière interne, c'est-à-dire par principe, à la position de l'observateur: il n'y a pas de point de vue performatif possible sur les causes, et la position de l'observateur n'implique donc pour les causes aucun déficit d'explication. En revanche, ce qui est principiellement barré à l'observateur, c'est de pouvoir donner les vraies raisons de l'action, car celles-ci sont nécessairement liés à la position performative de celui qui agit.

- La raison d'une action ne peut donc être fournie que par celui qui agit. La règle que l'on invoque alors ne résulte pas de la description d'une régularité, mais se réfère à l'adoption d'une convention; il s'agit d'une règle au sens de convention instituée, et par là même critiquable, en un sens où ne le sont pas les régularités.

14. Sur la base de ces premiers éléments théoriques, je propose les définitions suivantes: est ACTEUR celui pour qui la règle est une RAISON d'agir; est AGENT celui pour qui la règle agit comme CAUSE de son agir.

15. Cette caractérisation de l'acteur en fonction de son rapport à la règle opère un déplacement fondamental par rapport à ses caractérisations en termes de liberté. Poser le problème de l'acteur en termes de liberté, c'est nécessairement postuler une caractéristique ontologique de l'individu, celle d'être libre, qu'on définisse cette liberté en termes de droits, de pouvoir, de capacités, de faculté, de projet, d'existence, peu importe... On dote l'individu de la caractéristique d'être 
libre, puis on est inévitablement confronté au problème de ses limites (liberté individuelle / contraintes sociales). L'approche en termes de règles n'a pas ce problème ontologique, parce que l'individu y est d'emblée caractérisé pragmatiquement (et non ontologiquement), c'est-à-dire selon l'usage qu'il fait des règles et le rapport qu'il entretient avec elles. La question n'est pas de savoir si l'existence de telle règle contraint sa liberté ou pas, si cette règle lui laisse encore un espace de liberté et lequel, si l'individu se soumettant à telle règle peut encore être dit libre ou non, mais: quel usage fait-il des règles, quel type de rapport entretient-il avec elles? La seule capacité présupposée est celle de pouvoir gouverner son action par des règles, ce qui est la définition même de l'action; la définition ontologique de l'individu comme être de liberté est remplacée par sa définition pragmatique comme usager de règles. Il n'y a plus d'opposition systématique entre une aire de liberté et des contraintes sociales, mais une gamme d'usages possibles des règles qui structurent le monde qui nous entoure.

16. Mais ce double usage n'épuise pas la totalité des usages possibles de la règle. Lorsque l'on invente ou institue une règle (p.ex. une règle de droit, comme dans le cas des lois de bioéthique), on est auteur plus qu'acteur; lorsque l'on applique une règle préexistante, comme dans le cas du juge, on interprète la règle, avec toute la marge qu'il y a entre une application mécanique et l'évaluation par le jugement pratique (phronesis). La lecture d'un mode d'emploi laisse moins de marge interprétative (bien qu'elle ne soit pas nulle) que la décision juridique d'accorder des circonstances atténuantes; mais pour caractériser ces deux cas, il faut caractériser le type d'usage de la règle. Entre «l'acteur pur» et «l'agent pur», idéaux-types, il y a donc un continuum plus que des paliers nets; mais c'est à chaque fois le rapport à la règle qui en constituera la marque caractéristique. Il est vraisemblable par exemple que la posture la plus habituelle face aux règles sociales en général consiste à accepter la condition d' agent, donc à être un acteur qui se sait en position d'agent: ainsi le système salarial qui structure l'ensemble de notre mécanique sociale fait-il de la majorité des sociétaires des agents d'un système qu'ils n'ont jamais à proprement parler choisi, et que personne (aucune personne individuelle) n'a le pouvoir de réformer. Cela ne veut pas dire qu'il n'y a qu'une attitude possible face au système salarial, celle de la résignation stoïcienne: au contraire, il y a une foule d'attitudes intentionnelles possibles; par exemple, à titre indicatif :

- l'obéissance non questionnée face à ce qui est donc vécu sur le mode de la fatalité naturelle; on laisse alors agir les règles comme une cause aveugle;

- l'obéissance raisonnée, qui adhère passivement, mais en toute connaissance de cause, mue par exemple par la conviction qu'il s'agit là du meilleur système pour nous; on laisse alors agir les règles soit comme cause, si l'on pense simultanément que ces règles sont intangibles, soit comme raison, si l'on pense qu'il n'y a pas lieu de les modifier;

- la contestation passive, c'est-à-dire l'acceptation malgré soi d'un système de règles face auquel on se sent impuissant; on laisse alors agir les règles comme une cause hostile;

- la contestation ouverte, qui prône une modification des règles; les règles sont alors vécues comme des raisons qu'on n'a plus de bonnes raisons de suivre. 
En bref, c'est à chaque fois l'attitude intentionnelle face aux règles qui caractérise la position que l'on occupe sur le continuum qui va de l'agent à l'acteur. Sans doute, le maximum de la capacité d'acteur est-il activé lorsque l'on institue de nouvelles règles, alors que l'agent est à son comble d'agent lorsqu'il est dépendant à son insu de règles qu'il ne connaît pas. D'où le problème de la causalité inconsciente, que l'on rencontre de Freud à Searle.

17. Selon ce qui a été dit, on remarquera qu'une motivation inconsciente agit comme une détermination causale. Il y a là un problème essentiel pour les sciences humaines en général, c'est pourquoi il vaut la peine de le mentionner. Postuler l'existence d'un ensemble inconscient de règles gouvernant nos activités pour rendre compte de certaines régularités de comportement est à mon sens, d'un point de vue méthodologique, une étrange affaire, bien qu'exerçant indubitablement sur nos esprits un grand pouvoir d'attraction. La psychanalyse freudienne, mais aussi l'anthropologie structurale de Lévi-Strauss ou la sociologie de Bourdieu nous ont rendu familière l'idée générale d'une causalité inconsciente gouvernée par des règles «insues », c'est-à-dire échappant à la conscience des individus. Elles ne l'ont pas rendue claire pour autant. Sur la base de ce qui a été dit sur le lien interne qui reliait la position de l'observateur à la formulation d'hypothèses causales, on peut dire que le problème général récurrent à propos des règles inconscientes est le suivant: comment peut-on s'assurer qu'un système de règles présumées inconscientes offre l'explication pertinente du comportement considéré? Ou la seule? Ou la bonne? Le problème des règles inconscientes, c'est que dès qu'elles sont formulées, elles ne le sont plus, mais que tant qu'elles le restent, on ne peut pas savoir si on les suit. Et personne ne peut le savoir: ni l'observateur, qui n'a à sa disposition que des comportements manifestes, ni l'acteur, par définition. On ne peut donc pas le lui demander. C'est donc en toute cohérence de cause, si l'on peut dire, que ces mouvements de pensée en sont venus à nier la notion de sujet; plus rigoureusement, selon ce qui précède, on devrait dire qu'ils nient la capacité des sujets à être acteurs - c'est-à-dire mus par des raisons -, parce qu'ils sont toujours les agents de causes pour eux inconscientes: ils sont mus, à leur insu, par un autre qu'eux, un autre qu'eux qui ne peut par là même apparaître qu'à un observateur extérieur. Mais du coup, le théoricien - et, hélas, à l'inverse de la compréhension qu'il a de lui-même! - est mis en posture de ne pouvoir formuler que des conjectures invérifiables par nature, conjectures qui, dès lors qu'elles sont hypostasiées en réalité ultime, constituent ce qu'on peut assez rigoureusement appeler une idéologie. Cette posture méthodologique conduit à ce qu'on pourrait appeler une illusion causale, ou illusion de la causalité inconsciente, au sens où elle paraît offrir un principe explicatif de phénomènes définis, alors que ce principe même les soustraient par définition à toute vérification possible. (Si l'on voulait être suspicieux, on supposerait que c'est précisément parce qu'elles sont invérifiables que maints savants y recourent si volontiers.) Le caractère illusoire - ou idéologique - de ces «explications » tient selon moi à une approche insuffisamment critique de ce que veut dire «suivre une règle».

18. J'en viens maintenant aux considérations normatives que recèlent à mon sens les considérations qui précèdent. L'une des marques caractéristiques de la distinction entre régularités et conventions était la criticabilité de ces dernières. 
C'est pourquoi une recherche des ressources normatives d'une théorie critique de la règle peut prendre appui sur la notion de conflit - le conflit étant considéré dans son acception la plus large comme contestation d'une règle ${ }^{8}$. Ce qui importe ici plus spécifiquement, c'est que les règles à propos desquelles il y a conflit, étant nécessairement des règles instituées, sont donc toujours en quelque manière liées à ceux qui les utilisent, et en conséquence aux aspirations dont ils se réclament, et dont ils réclament reconnaissance: le fait même du conflit révèle la connexion interne qui relie les règles instituées aux aspirations des acteurs sociaux. Il est important dans ce contexte de parler d'aspirations plutôt que d'intérêts, parce que la notion large d'aspiration ne préjuge pas du type de raisons que l'on peut invoquer pour justifier une règle; ce que fait en revanche la notion d'intérêt, qui situe d'emblée l'enjeu d'un conflit, dans l'orbe de la concurrence utilitariste, ce qui est une inutile et fâcheuse réduction.

19. Or, cette connexion interne, systématique, entre aspirations et règles instituées recèle en tant que telle d'importantes ressources normatives. En effet, l'émergence factuelle des aspirations, dès lors qu'on peut la saisir sous la forme d'un conflit moral au moins potentiel, contient par elle-même un élément contrefactuel d'anticipation vers une communauté qui n'existe pas encore mais dont on aimerait qu'elle existât. Car appréhender ses propres aspirations comme entrant potentiellement en conflit avec les règles existantes, c'est déjà anticiper une communauté d'utilisateurs de la règle où ces aspirations, une fois reconnues, ne seraient précisément plus en conflit avec les règles de la communauté à laquelle on appartient. Mes aspirations se disent à l'indicatif, mais au moment où elles s'énoncent, leur reconnaissance est conditionnelle et ne peut encore être qu'anticipée contrefactuellement. Mais ce qui importe, c'est que l'émergence des aspirations, dès lors qu'elle est appréhendée comme un conflit avec les normes actuellement existantes, ne peut pas ne pas anticiper contrefactuellement la communauté qui reconnaîtrait ces aspirations, puisque c'est parce qu'elle ne les reconnaît pas encore qu'il y a émergence du conflit. Cette anticipation n'a donc à mon sens pas besoin de mobiliser le pathos de la fameuse communauté idéale de parole qu'évoquent Karl Otto Appel ou Jürgen Habermas dès lors que les normes entrent dans un processus de justification, avec toute la cathédrale normative qui s'ensuit; car ce n'est pas une communauté idéale de parole, même à titre de simple idéal régulateur, que l'on anticipe contrefactuellement lorsque l'on conteste la validité d'une règle, mais plus modestement un état de notre communauté tel qu'il n'existe pas encore. C'est bien là une anticipation contrefactuelle, mais qui ne fait pas derechef le saut conceptuel vers la communauté idéale de parole; juste le pas vers un état projeté, mais encore inexistant de notre communauté telle qu'on souhaiterait la voir advenir.

\footnotetext{
J'avais développé cette thèse dans La vertu du conflit, Paris, Cerf, 1995. La vertu du conflit était précisément de révéler, au sens quasi photographique du terme, la normativité des règles en litige; loin que les règles ne soient qu'un effet d'inculcation ou de violence symbolique (Bourdieu), le conflit à leur propos révèle la source de leur normativité. C'est pourquoi il peut y avoir une résolution normativement satisfaisante des conflits, c'est-à-dire une résolution qui ne soit pas que l'imposition du droit du plus fort.
} 
20. Mais du coup, l'on voit le lien qui relie les règles en litige aux aspirations des concernés. Car dès lors qu'un conflit est défini comme un conflit portant sur les règles, il met en litige les aspirations de ceux qui sont en quelque manière concernés par cette règle, et veulent désormais faire valoir ces aspirations. Les concernés sont les utilisateurs de la règle, sans quoi ils n'auraient pas de raison de déclencher un conflit à son propos. Ce qui veut dire que le conflit révèle les individus concernés dans leur capacité d'acteur, c'est-à-dire dans leur pouvoir d'action sur les règles qu'ils utilisent: ils ne veulent plus être les agents d'une règle qu'ils n'approuvent pas.

21. C'est aussi parce que les règles sont rattachées aux aspirations des concernés que ces règles sont plastiques, c'est-à-dire qu'elles sont susceptibles d'être modifiées. S'il n'y avait aucun lien entre les règles et les aspirations de ceux qui les utilisent, il n'y aurait aucune raison de déclencher des conflits à leur propos ni même de vouloir les modifier, aussi peu que cela fait sens de vouloir se révolter contre l'orbite des planètes. C'est donc dans une cohérence intime que se trouvent reliées les règles, les aspirations des utilisateurs des règles et la plasticité de cellesci, sans laquelle aucun conflit ne serait soluble de manière normativement satisfaisante, puisque si les règles ne l'étaient pas, il n'y aurait par définition aucune raison de vouloir les changer.

22. Ce lien interne entre règles instituées, acteurs sociaux et communauté anticipée dessine les contours d'un vaste programme politique dont on ne formulera ici que les principes directeurs. Car lier ainsi principiellement les règles instituées aux aspirations des concernés, c'est dire simultanément que c'est à eux de configurer leur espace normatif, c'est-à-dire le système de règles gouvernant leur coexistence. La démocratie apparaît donc comme le système où l'espace normatif appartient aux acteurs sociaux eux-mêmes, au sens où les utilisateurs des règles en sont aussi les auteurs, par quoi se trouve formulée une exigence générale, mais non triviale, de participation. Pour en revenir à une illustration mentionnée en introduction, on notera qu'il y a aujourd'hui un vaste mouvement politique de fond qui veut précisément faire accroire que les règles sociétales n'appartiennent pas aux acteurs sociaux qui les utilisent, voulant précisément les déposséder ainsi de leur espace de régulation au nom d'un fétichisme économique sans précédent - le fétichisme consistant justement à faire passer pour des régularités naturelles (le fonctionnement économique) ce qui est en réalité un ensemble de règles instituées.

23. Quoi qu'il en soit, on voit l'exigence procédurale de participation que révèle tout conflit à propos de règles instituées; l'exigence morale se concentre alors sur la capacité qu'a une communauté d'utilisateurs de la règle de prendre en compte l'expression des aspirations à propos de cette règle. C'est pourquoi on parle ici d'exigence procédurale: il s'agit d'une exigence de participation telle qu'elle se traduit dans une société pas sa capacité institutionnelle à assurer la meilleure participation de tous les concernés. Néanmoins, tout procédural qu'il soit, ce principe de participation, fondé sur une analyse de la règle et du conflit dont elle est susceptible, fait signe vers un principe de justice politique dont les termes peuvent s'énoncer comme suit: 
« EST RÉPUTÉE LÉGITIME TOUTE RÈGLE POUVANT ÊTRE ACCEPTÉE PAR LA RAISON PUBLIQUE AU TITRE DE LA RÉSOLUTION DU CONFLIT D'ASPIRATIONS DONT UNE RÈGLE LITIGIEUSE FAIT L'OBJET».

24. Par ce principe se trouvent traduits sur le plan normatif à la fois: la centralité de la règle, telle qu'elle peut être mise en évidence par une théorie du conflit, et qui déplace les termes de la problématique libérale classique d'une ontologie de l'individu vers une pragmatique de ses usages de la règle; le caractère public des règles litigieuses dont les critères d'appréciation en légitimité, fournis par la «raison publique», ne peuvent eux aussi être tirés que d'autres règles valant publiquement - analogiquement à la manière dont, chez Rawls, les critères du raisonnable sont fournis par un consensus par recoupement qui luimême est en accord avec ce qu'il appelle «la compréhension fondamentale de nous-mêmes ${ }^{9}$; le caractère productif des conflits, puisque c'est leur mérite que de problématiser la normativité de règles devenues litigieuses; et enfin, la connexion interne liant les acteurs sociaux aux règles de la régulation sociale, ce qui connecte le thème du conflit à celui de la reconnaissance: car en confrontant leurs aspirations et en voulant les faire publiquement reconnaître comme légitimes, les acteurs sociaux veulent se faire reconnaître en tant qu' ils sont porteurs de ces aspirations-là. En ce sens, les aspirations qu'ils expriment disent bien quelque chose d'eux-mêmes, de cette parcelle d'identité qu'expriment ces aspirations - c'est pourquoi le déni de reconnaissance qu'on peut opposer à une aspiration peut être vécu comme une marque de mépris. Pour le dire dans les termes qui ont été évoqués, le mépris consiste alors à nier chez l'individu sa capacité d'acteur, pour le réduire à sa simple condition d'agent.

Faculté des Lettres

Université de Genève

9 John Rawls, Justice et démocratie, trad. de Catherine Audard, Paris, Seuil, 1993, p. 78. 